\title{
Arteriolar biomechanics in a rat polycystic ovary syndrome model - Effects of parallel vitamin D3 treatment
}

\author{
L Sara ${ }^{1}$, GyL Nádasy ${ }^{2}$, P Antal ${ }^{2}$, M Szekeres ${ }^{3}$, A Monori-Kiss², EM Horváth ${ }^{2}$, \\ AM Tőkes ${ }^{4}$, G Masszi $^{5}$, E Monos ${ }^{2}$, Sz Várbirón ${ }^{1}$ \\ ${ }^{1} 2^{\text {nd }}$ Department Obstet. Gynecol., Faculty of Medicine, Semmelweis University, Budapest, Hungary \\ ${ }^{2}$ Institute of Human Physiology and Clinical Experimental Research, Faculty of Medicine, Semmelweis \\ University, Budapest, Hungary \\ ${ }^{3}$ Department of Physiology, Faculty of Medicine, Semmelweis University, Budapest, Hungary \\ ${ }^{4} 2^{\text {nd }}$ Department of Pathology, Faculty of Medicine, Semmelweis University, Budapest, Hungary \\ ${ }^{5}$ Department of Cardiology, Bajcsy-Zsilinszky Hospital, Budapest, Hungary
}

Received: January 12, 2012

Accepted after revision: April 24, 2012

To clarify the effects of dihydrotestosterone (DHT)-induced polycystic ovary syndrome (PCOS) on arteriolar biomechanics in a rat model and the possible modulatory role of vitamin $\mathrm{D}_{3}$. Methods and Results: The PCOS model was induced in female Wistar rats by ten-weeks DHT treatment. Arteriolar biomechanics was tested in arterioles by pressure arteriography in control as well as DHT- and DHT with vitamin $\mathrm{D}_{3}$-treated animals in contracted and passive conditions. Increased wall stress and distensibility as well as increased vascular lumen were detected after DHT treatment. Concomitant vitamin $\mathrm{D}_{3}$ treatment lowered the mechanical load of the arterioles and restored the vascular diameter. Conclusion: The hyperandrogenic state resulted in more rigid, less flexible arteriolar walls with increased vascular lumen compared with controls. DHT treatment caused eutrophic remodelling of gracilis arteriole. These prehypertensive alterations caused by chronic DHT treatment were mostly reversed by concomitant vitamin $\mathrm{D}_{3}$ administration.

Keywords: PCOS, rat model, dihydrotestosterone, arteriolar biomechanics, vitamin D

Polycystic ovary syndrome is a frequent cause of infertility and affects 5-8 percents of women. It is also well known that in PCOS, women of reproductive age may develop vascular damage. Ten years ago, Lakhani and Hardiman showed that the internal carotid artery pulsatility index was decreased and cardiovascular risk was increased in women with PCOS (3). The mechanisms behind this increased risk and the possible therapeutic approaches are still in the focus of research. Women with PCOS have a high prevalence of early-onset atherosclerosis, metabolic syndrome and insulin resistance, and they might develop hypertension during their reproductive period (2). These abnormalities are partly explained by biomechanical remodelling of resistance arteries. The aim of our study was to identify early mechanical alterations in a morphologically stable skeletal muscle vessel, the gracilis

Corresponding authors: Szabolcs Várbiró*, MD, PhD and Levente Sara**, MD

$2^{\text {nd }}$ Department Obstet. Gynecol., Faculty of Medicine, Semmelweis University

Üllői út 78/A, H-1083 Budapest, Hungary

*Phone: +36/20/3359099; Fax: +36/1/3137856; E-mail: varbiroszabolcs@gmail.com

**Phone: +36/70/3815681; Fax: +36/1/2100290; E-mail: saralevente@gmail.com 
arteriole, in a rat PCOS model. Manneras et al. have developed an adequate PCOS rodent model. Adolescent female rats that receive chronic dihydrotestosterone (DHT) treatment, develop a hyperandrogenic condition similar to human $\operatorname{PCOS}(6,18)$.

In clinical practice, chronic treatment of PCOS might improve mechanical properties of arteries. The positive effect from insulin-sensitising therapy on the mechanical and pharmacological responsiveness of arteries has recently been shown $(1,8)$. Vitamin $\mathrm{D}_{3}$ may also be used as adjuvant therapy in women with PCOS (14). The positive effect of vitamin $\mathrm{D}_{3}$ therapy on the prevention of general cardiovascular complications has been demonstrated $(9$, 17). Herein, we investigated the effect of low-dose vitamin $D_{3}$ treatment on the vascular biomechanical adaptation of gracilis arterioles in a rat PCOS model.

\section{Methods and Materials}

\section{Chemicals}

Pentobarbital (Nembutal, Phylaxia-Sanofi, Budapest, Hungary) was used for anesthesia. Following chronic surgical intervention, $20 \mathrm{mg}$ amoxicillin and $4 \mathrm{mg}$ clavulanic acid (Augmentin GlaxoSmithKline, Memphis, US) in $0.2 \mathrm{ml}$ saline was administered intramuscularly to prevent infection. We followed the protocol to induce experimental polycystic ovary syndrome described by Manneras et al. (6). Continuous-release pellets that contained $7.5 \mathrm{mg}$ dihydrotestosterone (DHT, Innovative Research of America, Sarasota, Fl, USA) were applied for 70 days. Eighty-three micrograms/day release for 90 days is guaranteed by the manufacturer. We purchased $1.25(\mathrm{OH})_{2} \mathrm{D}_{3}$ vitamin (Inj. Calcijex, $2 \mu \mathrm{g} / \mathrm{ml}$ ) from Abbott Lab., Illinois, USA. The composition of the normal Krebs-Ringer solution used in these in vitro studies was as follows (in mmol/L): $\mathrm{NaCl} 119, \mathrm{KCl} 4.7, \mathrm{NaH}_{2} \mathrm{PO} 41.2, \mathrm{MgSO}_{4}$ 1.17, $\mathrm{NaHCO}_{3} 24, \mathrm{CaCl}_{2} 2.5$, glucose 5.5 and EDTA 0.034. The $\mathrm{Ca}^{2+}$-free Krebs solution that was used to relax the smooth muscle contained the following (in mmol/L): $\mathrm{NaCl} 92, \mathrm{KCl} 4.7$, $\mathrm{NaH}_{2} \mathrm{PO}_{4} 1.18, \mathrm{MgCl}_{2} 20, \mathrm{MgSO}_{4} 1.17, \mathrm{NaHCO}_{3} 24$, glucose 5.5, EGTA 2 and EDTA 0.025. The temperature of the solution was maintained at $37^{\circ} \mathrm{C}$, and it was bubbled with $5 \% \mathrm{CO}_{2}$, $20 \% \mathrm{O}_{2}$, and $75 \% \mathrm{~N}_{2}$, which stabilized the $\mathrm{pH}$ at 7.4. Norepinephrine (NE), was obtained from Sigma-Aldrich Co. (St. Louis, MO, USA and Budapest, Hungary) and freshly prepared on the day of the experiment.

\section{Animals}

Thirty adolescent (21- to 28-day-old) female Wistar rats (provided by the Animal Facility at the Semmelweis University in agreement with Charles River Ltd.) that weighed 100-140 g when the study began were used. Twenty of the animals received subcutaneous pellets containing $7.5 \mathrm{mg}$ DHT implanted under the back skin under anesthesia (Nembutal $45 \mathrm{mg} / \mathrm{kg}$ i.p.) in sterile conditions (DHT-treated female groups), which induces polycystic ovary syndrome (6). Ten animals underwent sham operations. During and after these procedures there were no surgical complications, no signs of distress or suffering.

Ten DHT-treated animals received $120 \mathrm{ng} / 100 \mathrm{~g}$ body weight/ week $1.25(\mathrm{OH})_{2} \mathrm{D}_{3}$ vitamin (DHT+D3 group) subcutaneously as previously described by Przybilski et al. We applied a weekly dosage instead of a daily administration(9) to reduce stress on the animals. A vehicle was given to the remaining 20 animals. No medical or surgical complications were observed. Conventional rat food and tap water were provided ad libitum. After eight weeks of DHT treatment a significantly higher 120-minute postload insulin level were measured in DHT group than in the controls, and this difference was eliminated by the $\mathrm{D}_{3}$ vitamin treatment 
(10). There was not any difference in glucose levels (10). The investigation conforms to the Principles of Laboratory Animal Care NIH publication No. 85-23, revised 1985) as well as the Euroconform Hungarian Law on Animal Care (XXVIII/1998), and the study protocol was accepted by the institutional Animal Care Commission (IRB approval: 22.1/2960/ 003/2009).

\section{Biomechanics of a musculocutaneous arteriole (pressure arteriography)}

The animals' blood pressure was measured directly by cannulation of the carotid artery in general surgical anesthesia. Nembutal was given i.p., $50 \mathrm{mg} / \mathrm{kg}, 1 / 3 \mathrm{rd}$ of the dose repeated if repeated checks of pain and corneal reflexes made it desirable. Then the animals were bled under anesthesia, adductor muscle group and ovaries were removed from the cadavers. After opening the iliofemoral region, the arteriole, which had an in vivo diameter of approximately $200 \mu \mathrm{m}$ and is the blood supply for the gracilis muscle, was removed and placed into a vessel chamber filled with normal Krebs-Ringer (nKR) solution. It was cannulated at both ends with plastic microcannulas and extended to its in vivo length. Both cannulas were connected to pressure-servo pumps (Living Systems, Burlington, VT), and the arterioles were pressurised under a no-flow condition to $50 \mathrm{mmHg}$ intraluminal pressure.

The outer and the inner diameters of the arterioles were measured by video-microscopic pressure microangiometry. In this setup, the glass-bottomed tissue bath was positioned in the light path of an inversed Leica microscope. A magnified picture of the vessel was formed with the aid of a video camera (Leica DFC320) and Leica QWin software. The digitalized pictures were saved, and off-line measurements of the inner and outer diameter were made using Leica QWin image analysis software.

The gracilis arterioles were allowed to equilibrate for 30 minutes at $50 \mathrm{mmHg}$ intraluminal pressure in an oxygenised nKR solution. Following incubation, the pressure was decreased to $10 \mathrm{mmHg}$ and then increased to $100 \mathrm{mmHg}$ in $10-\mathrm{mmHg}$ pressure steps. The steady state diameter at each step was measured. During NE contraction (after 10-minute incubation with $10^{-6} \mathrm{M} \mathrm{NE}$ ) the pressure-diameter curve was repeated as described above. After recording the NE-curve, the passive diameter was measured in $\mathrm{Ca}^{2+}$-free Krebs solution. Arteriolar segments were incubated for 20 minutes, and the steady-state, fully relaxed diameter was then measured as described above. We used a micrometer etalon (Wild) for the calibrations.

\section{Biomechanical calculations}

From the originally calibrated pressure-diameter plots, the following geometrical and biomechanical parameters were computed for each intraluminal pressure level(16). Tangential stress was computed according to the Laplace equation: $\sigma_{\text {tang }}=\mathrm{P} \times \mathrm{r}_{\mathrm{i}} / \mathrm{h}$, where $\sigma_{\text {tang }}$ is the tangential (circumferential) wall stress, $\mathrm{p}$ is the intraluminal pressure, $\mathrm{r}_{\mathrm{i}}$ is the inner radius and $h$ is the wall thickness ( $h=r_{o}-r_{i}$, where $r_{o}$ is the outer radius). Incremental distensibility was computed as $\mathrm{D}_{\text {inc }}=\Delta \mathrm{V} / \mathrm{V} \times \Delta \mathrm{P}$ where $\mathrm{D}_{\text {inc }}$ is the incremental distensibility and $\Delta \mathrm{V}$ is the change in vessel lumen volume in relation to the initial volume $\mathrm{V}$ in response to the pressure change $\Delta \mathrm{P}$. The circumferential incremental elastic modulus was computed from the following equation: $\mathrm{E}_{\text {inc }}=\left(\Delta \mathrm{P} / \Delta \mathrm{r}_{\mathrm{o}}\right) \times 2 \mathrm{r}_{\mathrm{i}}^{2} \times \mathrm{r}_{\mathrm{o}} /\left(\mathrm{r}_{\mathrm{o}}^{2}-\mathrm{r}_{\mathrm{i}}^{2}\right)$, where $\mathrm{E}_{\text {inc }}$ is the incremental elastic modulus, $\mathrm{r}_{\mathrm{i}}$ is the inner radius, $r_{o}$ is the outer radius, and $\Delta r_{o}$ is the change in outer radius in response to intraluminal pressure change of $\Delta \mathrm{P}$. 


\section{Histology}

The ovaries and arterioles were formaldehyde fixed, stained with hematoxylin-eosine and histologically examined by using the Pannoramic Viewer software. We detected a polycystic morphology in the DHT-treated groups and normal ovaries in the controls, as published earlier by Manneras and Yanes $(6,10,18)$.

\section{Statistical analysis}

For statistical analysis, repeated-measures ANOVA was used. In vitro parameters were plotted as a function of intraluminal pressure, and the curves were analysed by paired comparisons of the treatment groups. One-way ANOVA was applied for the discrete parameters (e.g., blood pressure). As post hoc test, Tukey`s test was used. $p<0.05$ was uniformly accepted as a significant difference. Data are presented as mean \pm SEM. GraphPad Prism version $5.0 \mathrm{~d}$ was used as statistical software.

\section{Results}

\section{Physiological parameters}

The mean arterial pressure was $122 \pm 3 \mathrm{mmHg}, 123 \pm 6 \mathrm{mmHg}$ and $123 \pm 4 \mathrm{mmHg}$ for the controls, the DHT-group and the DHT+vitamin $\mathrm{D}_{3}$-treated group, respectively (nonsignificant). The body weights at the end of the experiment were $298 \pm 8 \mathrm{~g}, 354 \pm 16 \mathrm{~g}$ and $353 \pm 9 \mathrm{~g}$ for the same groups, respectively. The DHT-treated animals had significantly higher body weights than the controls $(p<0.01)$, but there was no difference between the two DHTtreated groups.

\section{Arteriole geometry}

DHT treatment increased the relaxed outer (Fig.1) radii (measured in a $\mathrm{Ca}^{2+}$-free medium), which were diminished by a parallel vitamin $\mathrm{D}_{3}$ treatment $\left(p<0.05\right.$ for DHT vs. DHT $+\mathrm{D}_{3}$ comparison, Fig. 1). The wall thicknesses for the different groups in the $\mathrm{Ca}^{2+}$-free medium were as follows: $20 \pm 2 \mu \mathrm{m}, 19 \pm 1 \mu \mathrm{m}$ and $20 \pm 1 \mu \mathrm{m}$ in the control, DHT- and DHT + vitamin $\mathrm{D}_{3}$-treated groups, respectively (as measured at $50 \mathrm{mmHg}$, non-significant). In $\mathrm{Ca}^{2+}$ free solution, the cross section areas for the vessel walls were $12080 \pm 1627 \mu \mathrm{m}^{2}, 12542 \pm$ $1090 \mu \mathrm{m}^{2}$ and $11348 \pm 660 \mu \mathrm{m}^{2}$ for these groups, which were not significant.

\section{Arteriole elasticity}

The mechanical load on the vessel wall was measured, and the tangential wall stress was significantly higher throughout the entire pressure range for the DHT-treated group compared with the other two groups in both $\mathrm{Ca}^{2+}$-free solution and during NE contraction (Fig. 2a, b, $p$ $<0.05$ ). Vitamin $\mathrm{D}_{3}$ treatment induced a partial reversal in the NE-contracted segments and an overcompensation in the $\mathrm{Ca}^{2+}$-free solution (Fig. 2, both for DHT vs. DHT + D3 and the control vs. DHT $+\mathrm{D} 3, p<0.05)$.

Isobaric distensibility was significantly higher throughout the entire pressure range for the DHT group compared with the other two groups in both the $\mathrm{Ca}^{2+}$-free medium and during norepinephrine contraction (Fig. 3a, b, $p<0.05$ ). In response to a parallel treatment with vitamin $\mathrm{D}_{3}$, relaxed distensibility fully returned to the control value (Fig. 3a), while NE contraction only generated a partial recovery (Fig. 3b). 


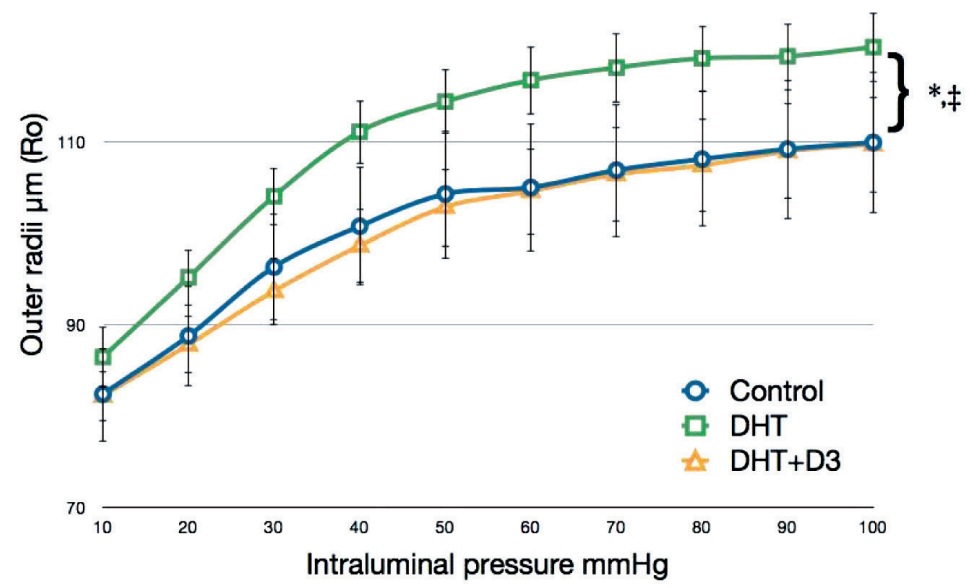

Fig. 1. Outer radii (Ro) in a $\mathrm{Ca}^{2+}$-free solution.

The outer radii from $\mathrm{Ca}^{2+}$-free relaxation at $\mathrm{P}=50 \mathrm{mmHg}$ were not different in the controls and DHT $+\mathrm{D} 3$. Both radii were smaller than in the arterioles isolated from the DHT-treated animals $(p<0.05)$. The values are expressed as the mean \pm SEM. *DHT was significantly different from the control. $\$$ The DHT $+\mathrm{D} 3$ was significantly different from the DHT group
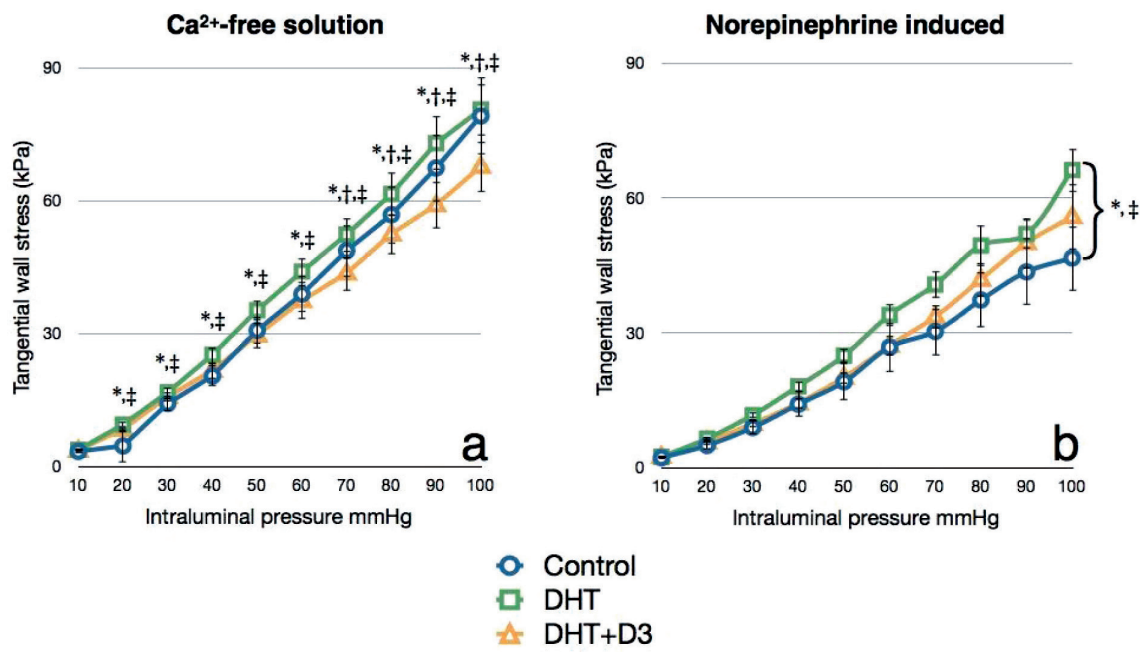

Fig. 2a. Tangential wall stress in the $\mathrm{Ca}^{2+}$-free solution.

The tangential wall stress was significantly greater in the DHT group than in the other two groups throughout the entire pressure range, $(p<0.001)$. Between $70-100 \mathrm{mmHg}$, the stress was significantly lower in the DHT $+\mathrm{D} 3$ group $(p<0.001)$.

Fig. 2b. NE-induced tangential stress. Tangential wall stress was significantly greater in the DHT group than in the other two groups throughout the entire pressure range, $(p<0.001)$.

Values are expressed as the mean \pm SEM. * DHT was significantly different from the control. $\uparrow$ DHT + D3 was significantly different from the control. $\$$ The DHT + D3 group was significantly different from the DHT group 
$\mathrm{Ca}^{2+-}$-free solution

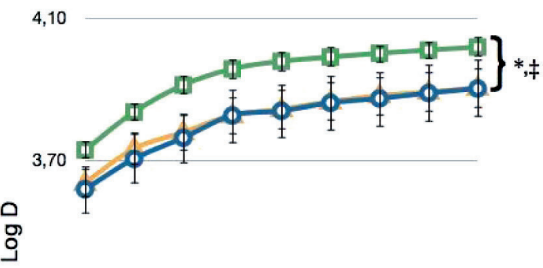

3,30

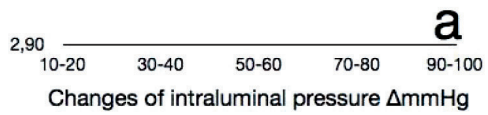

\section{Norepinephrine induced}

4,10

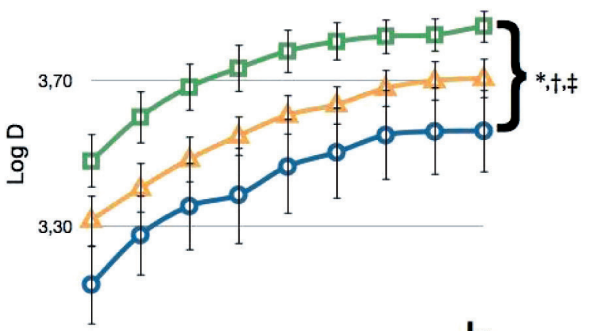

b

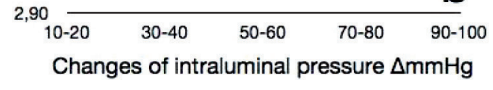

Changes of intraluminal pressure $\Delta \mathrm{mmHg}$

\section{- Control \\ DHT \\ DHT+D3}

Fig. 3a. Distensibility in a $\mathrm{Ca}^{2+}$-free solution. Distensibility was significantly greater in the DHT group compared with the other two groups throughout the entire pressure range $(10-100 \mathrm{mmHg}),(p<0.0001)$.

Fig. 3b. NE induced distensibility. Each group was significantly different throughout the entire pressure range $(p<0.0001)$. Distensibility was greatest in the DHT animals and lowest in the control animals.

Values are expressed as the mean \pm SEM. * DHT was significantly different from the control. $\uparrow$ DHT + D3 was significantly different from the control. $\$$ The DHT + D3 group was significantly different from the DHT group

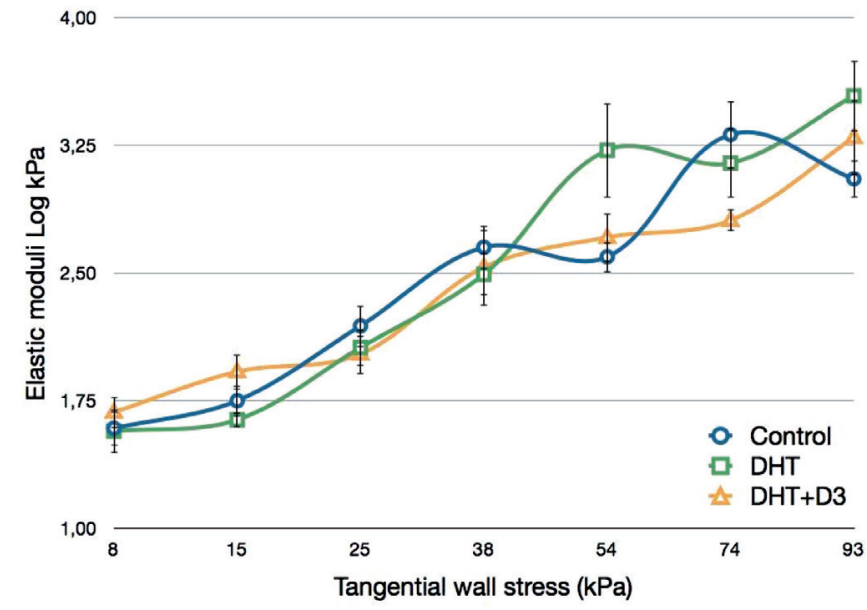

Fig. 4. Elastic moduli as a function of tangential wall stress in the $\mathrm{Ca}^{2+}$-free solution. The values for the abscissa show the elastic moduli $(\operatorname{logkPa})$ for the arterioles from the control rats as well as the DHT- and DHT + D3-treated rats. The ordinata shows the tangential stress $(\mathrm{kPa})$. There were no significant differences between the groups. The values are expressed as the mean \pm SEM 
Wall elastic moduli were not affected by DHT and vitamin $\mathrm{D}_{3}$ treatment (in a $\mathrm{Ca}^{2+}$-free solution, the $\log$ elastic moduli were $2.8 \pm 0.14 \log (\mathrm{kPa}), 2.7 \pm 0.16 \log (\mathrm{kPa})$ and $2.4 \pm 0.11$ $\log (\mathrm{kPa})$ in the control, DHT- and DHT + vitamin $\mathrm{D}_{3}$-treated groups, respectively, ns.). There was no difference in the elastic moduli as a function of tangential wall stress (Fig. 4, ns.).

\section{Discussion}

Chronic DHT treatment of female rats, which is an animal model for human PCOS, induced significant alterations in the biomechanical properties of a skeletal muscle arteriole. The relaxed lumen was dilated but this was not accompanied by an elevation in wall mass (eutrophic remodelling). This elevated wall stress was accompanied by an increase in isobaric distensibility. The elastic moduli did not change, which demonstrates that passive elastic element remodelling was limited.

Vitamin $\mathrm{D}_{3}$ is an adjuvant therapy for PCOS (14), and the cardiovascular protective effect of low-dose vitamin $\mathrm{D}_{3}$ therapy is widely accepted $(9,17,19)$. Our experiments demonstrate that Vitamin $\mathrm{D}_{3}$ can reverse morphological remodelling, elevation of wall stress and distensibility in a resistance vessel.

This is the first study to explore the details of vascular biomechanical adaptation of a resistance artery in an experimental PCOS model. We are the first to show that these changes are partially counteracted by vitamin $\mathrm{D}_{3}$, which is as adjuvant treatment often applied for PCOS.

Our results show basic changes in vascular biomechanics in response to DHT treatment; the arteriolar walls become more rigid with increased tangential wall stress compared with the controls. Thus, the mechanical load of the vessels increases. Under passive circumstances, vitamin $\mathrm{D}_{3}$ treatment fully restores the biomechanical balance of the vessel wall. However, in response to norepinephrine, mechanical load of the vessel wall increases compared with the control group, although it does not reach the level of the DHT-treated group.

In our study, we used an experimental PCOS model described by Manneras. The DHT treatment scheme used herein has proved to be an adequate rat PCOS model (6).

It has been shown that an 8- to 12-week DHT treatment - identical to the treatment that we used - induces polycystic ovary syndrome and significantly (3-fold) increases androgen levels in female rats (6). Yanes detected several metabolic abnormalities (18). During a 90day treatment, she detected an increase in the rat spontaneous proteinuria; morphological renal damage; elevated TGF alpha; leptin and cholesterol levels. These metabolic abnormalities might also influence arterial mechanics; distensibility and stiffness. Despite the normal estradiol level, DHT-treated animals exhibited oestrus cycle dysfunction $(6,18)$. A significant increase in body weight was detected, which was also noted in herein. This effect was not influenced by vitamin $\mathrm{D}_{3}$ treatment.

The DHT treatment applied by Yanes et al. caused hypertension when it was administered for a longer period of time (90 days). During our 70-day treatment, no significant differences were found between the blood pressure values of the experimental groups. However, the tangential wall stress and distensibility differences that were detected even in normotensive animals might be considered prehypertensive abnormalities. The enhanced mechanical load of the arteriolar wall is the local factor for the self-perpetuating, vicious cycle of emerging hypertension. In the vitamin D-treated group, tangential wall stress is not elevated - this 
might be a local effect against development of hypertension. This effect of vitamin D might be explained by enhanced proliferation of elastin components as it was demonstrated in aortic smooth muscle cells in vitro (15).

Because the blood pressures for the different animal groups did not differ in our study, the vascular biomechanical changes detected are directly attributable to either a hyperandrogenic state or induced hyperinsulinemia/insulin resistance. As we reported previously, insulin resistance was developed after eight weeks of DHT treatment (10). It is well known that vascular biomechanical functions are worse in PCOS. In 2000, Lakhani suggested that internal carotid artery pulsatility index was decreased, and cardiovascular risk is increased in women with PCOS (3). Lakhani then showed an increased intima-media thickness for the common carotid and femoral arteries in young (under the age of 35) women with PCOS (4). It is known that in PCOS, large arteries become less elastic and more rigid; the stiffness and intima-media thickness of the common carotid artery increases, and its distensibility decreases $(2,5,12)$. These changes correlate with a hyperandrogenic state rather than obesity (5). Calcification of the coronary arteries is enhanced (2), and a higher brachial-ankle pulse wave velocity is detected (11); thus, the vicious cycle of atherosclerosis also begins at an earlier age. Therefore, the early development of atherosclerosis and increased cardiovascular risk can be regarded as consequences of the hyperandrogenic state $(2,11)$. According to Soares, the early increase of cardiovascular risk, which is independent of other risk factors, is a part of polycystic ovary syndrome (12). Meyer has suggested that in addition to hyperandrogenic symptoms, changes in glucose metabolism and arterial stiffness (pulse wave velocity) should also be considered in treating PCOS. Meyer suggested treatment with metformin instead of high-dose oral contraceptives (7). Agarwal has shown that metformin decreases arterial stiffness, aortic and brachial pulse wave velocity, and aortic augmentation index (1). In PCOS, vascular biomechanical damage is essential and progresses faster than in the general population (2). The treatment modalities used in PCOS often modify this risk (1, 7). High-dose oral contraceptives might increase cardiovascular risk; metformin has been shown to decrease this risk, whereas low-dose oral contraceptives likely have no significant effect $(1,7)$. Herein, we provide these first data for the effect of vitamin $\mathrm{D}_{3}$ on the mechanical properties of arteries in PCOS. Vitamin $\mathrm{D}_{3}$ can be administered as an adjuvant therapy for PCOS (14), and the cardiovascular protective effect of low-dose vitamin $\mathrm{D}_{3}$ therapy is widely known $(9,17,19)$. The results from our study show that for PCOS, vitamin D is an adjuvant treatment that might inhibit arterial vascular mechanical impairment and potentiate the vascular protective effect of metformin because of its distinct mechanism.

Chronic vitamin $\mathrm{D}_{3}$ treatment directly decreased blood pressure and the vasoconstrictor response in isolated arteries from SHR rats. The latter effect was attributed to reduced COX1 expression, while intracellular Ca-levels remained unchanged(17). Other direct effects of vitamin D on vasodilation were also suspected which are independent from Ca metabolism (13). Vitamin $\mathrm{D}_{3}$ treatment mostly prevented arteriolar morphological remodelling and the increase in lumen volume caused by DHT treatment.

\section{Conclusions}

This is the first study to investigate the details of the vascular biomechanical adaptation of skeletal muscle arterioles in a PCOS model. Our results show that the hyperandrogenic state led to more rigid, less flexible arteriolar walls. In the relaxed vessels, vitamin $\mathrm{D}_{3}$ treatment corrected wall distensibility and mechanics to the control level. However, NE contraction 
resulted in slightly less flexible vessels (Figs 2,3). In conclusion, pathological prehypertensive biomechanical adaptation of arterioles was found in this PCOS model that was mostly prevented by concomitant vitamin $\mathrm{D}_{3}$ administration.

\section{Acknowledgements}

The authors wish to express their gratitude to Mrs. Ildikó Murányi for her devoted efforts in solving the technical problems in the laboratory work. This study was sponsored by a research grant from the Hungarian Society of Hypertension. Financial support: Hungarian Society of Hypertension.

\section{REFERENCES}

1. Agarwal N, Rice SP, Bolusani H, Luzio SD, Dunseath G, Ludgate M, Rees DA: Metformin reduces arterial stiffness and improves endothelial function in young women with polycystic ovary syndrome: a randomized, placebo-controlled, crossover trial. J. Clin. Endocrinol. Metab. 95, 722-730 (2010)

2. Dokras A: Cardiovascular disease risk factors in polycystic ovary syndrome. Semin. Reprod. Med. 26, 39-44 (2008)

3. Lakhani K, Constantinovici N, Purcell WM, Fernando R, Hardiman P: Internal carotid artery haemodynamics in women with polycystic ovaries. Clin. Sci. (Lond). 98, 661-665 (2000)

4. Lakhani K, Hardiman P, Seifalian AM: Intima-media thickness of elastic and muscular arteries of young women with polycystic ovaries. Atherosclerosis 175, 353-359 (2004)

5. Luque-Ramírez M, Mendieta-Azcona C, Alvarez-Blasco F, Escobar-Morreale HF: Androgen excess is associated with the increased carotid intima-media thickness observed in young women with polycystic ovary syndrome. Hum. Reprod. 22, 3197-3203 (2007)

6. Mannerås L, Cajander S, Holmang A, Seleskovic Z, Lystig T, Lonn M, Stener-Victorin E: A new rat model exhibiting both ovarian and metabolic characteristics of polycystic ovary syndrome. Endocrinology 148, 37813791 (2007)

7. Meyer C, McGrath BP, Teede HJ: Effects of medical therapy on insulin resistance and the cardiovascular system in polycystic ovary syndrome. Diabetes Care. 30, 471-478 (2007)

8. Naka KK, Kalantaridou SN, Kravariti M, Bechlioulis A, Kazakos N, Calis KA, Makrigiannakis A, Katsouras CS, Chrousos GP, Tsatsoulis A, Michalis LK: Effect of the insulin sensitizers metformin and pioglitazone on endothelial function in young women with polycystic ovary syndrome: a prospective randomized study. Fertil. Steril. 95, 203-209 (2011)

9. Przybylski R, Mccune S, Hollis B, Simpson RU: Vitamin D deficiency in the spontaneously hypertensive heart failure $[\mathrm{SHHF}]$ prone rat. Nutr. Metab. Cardiovasc. Dis. 20, 641-646 (2010)

10. Sara L, Antal P, Masszi G, Buday A, Horváth EM, Hamar P, Monos E, Nádasy GL, Várbiró S: Arteriolar insulin resistance in a rat model of polycystic ovary syndrome. Fertil. Steril. 97, 462-468 (2012)

11. Sasaki A, Emi Y, Matsuda M, Sharula, Kamada Y, Chekir C, Hiramatsu Y, Nakatsuka M: Increased arterial stiffness in mildly-hypertensive women with polycystic ovary syndrome. J. Obstet. Gynaecol. Res. 37, 402-411 (2011)

12. Soares GM, Vieira CS, Martins WP, Franceschini SA, dos Reis RM, Silva de Sá MF, Ferriani RA: Increased arterial stiffness in nonobese women with polycystic ovary syndrome (PCOS) without comorbidities: one more characteristic inherent to the syndrome? Clin. Endocrinol. (Oxf). 71, 406-411 (2009)

13. Tare M, Emmett SJ, Coleman HA, Skordilis C, Eyles DW, Morley R et al.: Vitamin D insufficiency is associated with impaired vascular endothelial and smooth muscle function and hypertension in young rats. J. Physiol. 589, 4777-4786 (2011)

14. Thys-Jacobs S, Donovan D, Papadopoulos A, Sarrel P, Bilezikian JP: Vitamin D and calcium dysregulation in the polycystic ovarian syndrome. Steroids 64, 430-435 (1999)

15. Tukaj C: Enhanced proliferation of aortal smooth muscle cells treated by 1,25(OH)2D3 in vitro coincides with impaired formation of elastic fibres. Int. J. Exp. Pathol. 89, 117-24 (2008)

16. Várbíró S, Nádasy GL, Monos E, Vajó Z, Ács N, Miklós Z, Tökés AM, Székács B: Effect of ovariectomy and hormone replacement therapy on small artery biomechanics in angiotensin-induced hypertension in rats. J. Hypertens. 18, 1587-1595 (2000) 
17. Wong MS, Delansorne R, Man RY, Svenningsen P, Vanhoutte PM: Chronic treatment with vitamin D lowers arterial blood pressure and reduces endothelium-dependent contractions in the aorta of the spontaneously hypertensive rat. Am. J. Physiol. 299, H1226-1234 (2010)

18. Yanes LL, Romero DG, Moulana M, Lima R, Davis DD, Zhang H, Racusen LC, Reckelhoff JF: Cardiovascularrenal and metabolic characterization of a rat model of polycystic ovary syndrome. Gender Med. 8, 103-115 (2011)

19. Yiu YF, Chan YH, Yiu KH, Siu CW, Li SW, Wong LY, Lee SW, Tam S, Wong EW, Cheung BM, Tse HF: Vitamin D deficiency is associated with depletion of circulating endothelial progenitor cells and endothelial dysfunction in patients with type 2 diabetes. J. Clin. Endocrinol. Metab. 96, E830-835 (2011) 